\title{
ACCRETION OF ROCKY PLANETS BY HOT JUPITERS
}

\author{
Jacob A. Ketchum ${ }^{1}$, Fred C. Adams ${ }^{1,2}$, and Anthony M. Bloch ${ }^{1,3}$ \\ ${ }^{1}$ Michigan Center for Theoretical Physics, Physics Department, University of Michigan, Ann Arbor, MI 48109, USA \\ ${ }^{2}$ Astronomy Department, University of Michigan, Ann Arbor, MI 48109, USA \\ ${ }^{3}$ Department of Mathematics, University of Michigan, Ann Arbor, MI 48109, USA \\ Received 2011 August 9; accepted 2011 September 21; published 2011 October 7
}

\begin{abstract}
The observed population of Hot Jupiters displays a stunning variety of physical properties, including a wide range of densities and core sizes for a given planetary mass. Motivated by the observational sample, this Letter studies the accretion of rocky planets by Hot Jupiters, after the Jovian planets have finished their principal migration epoch and become parked in $\sim 4$ day orbits. In this scenario, rocky planets form later and then migrate inward due to torques from the remaining circumstellar disk, which also damps the orbital eccentricity. This mechanism thus represents one possible channel for increasing the core masses and metallicities of Hot Jupiters. This Letter determines probabilities for the possible end states for the rocky planet: collisions with the Jovian planets, accretion onto the star, ejection from the system, and long-term survival of both planets. These probabilities depend on the mass of the Jovian planet and its starting orbital eccentricity, as well as the eccentricity damping rate for the rocky planet. Since these systems are highly chaotic, a large ensemble $\left(N \sim 10^{3}\right)$ of simulations with effectively equivalent starting conditions is required. Planetary collisions are common when the eccentricity damping rate is sufficiently low, but are rare otherwise. For systems that experience planetary collisions, this work determines the distributions of impact velocities - both speeds and impact parameters - for the collisions. These velocity distributions help determine the consequences of the impacts, e.g., where energy and heavy elements are deposited within the giant planets.
\end{abstract}

Key words: planet-disk interactions - planets and satellites: dynamical evolution and stability - planets and satellites: formation

Online-only material: color figures

\section{INTRODUCTION}

With hundreds of alien worlds detected, extrasolar planets have dramatically completed their migration into the mainstream of astronomy. The initial discoveries (Mayor \& Queloz 1995; Marcy \& Butler 1996) showed that the orbital elements of extrasolar planets are significantly different from those of solar system planets. Some giant planets are found in short-period or$\operatorname{bits}\left(P_{\text {orb }} \approx 4\right.$ days; semi-major axes $a \approx 0.05 \mathrm{AU}$ ), while others have longer orbits with a range of eccentricities, $0 \leqslant e \leqslant 0.9$. Subsequent discoveries indicate that such planetary systems are common and display a rich variety of architectures (Marcy \& Butler 2000; Hatzes et al. 2000; Perryman 2000; Udry et al. 2007). The galactic planetary census is growing rapidly, and we can probe their physical properties, dynamics, composition, and even their weather.

An important subset of migrating Jovian planets reaches the inner edge of their parental disks, where they enter orbits with periods $P_{\text {orb }} \sim 2-5$ days. Much of our knowledge regarding the physical properties of extrasolar planets comes from this population, primarily those planets observed in transit. Observations of these transiting planets have driven an exploration of the planetary mass-radius relation, which shows several unexpected features. The mass distribution of these planets is wide, spanning more than three decades. The distribution of inferred densities ranges over two orders of magnitude, with $\rho \approx 0.16-26 \mathrm{~g} \mathrm{~cm}^{-3}$. Extrasolar planets thus span a wide range of radii for a given mass. The mass-radius relation for Hot Jupiters depends on many factors, including metallicity, core mass, stellar irradiation, and additional heat sources (Bodenheimer et al. 2003, hereafter BLL; Laughlin et al. 2011).

This Letter explores one channel for Jovian planets to change their structure after reaching the stellar vicinity: Hot Jupiters can accrete additional rocky bodies while they are parked in close orbits. This accretion process increases the planetary mass, core mass, metallicity, and density of the Jovian target. This scenario works as follows: Jovian planets stop their inward migration at semi-major axes corresponding to $\sim 4$ day orbital periods. Although the reason for planets halting their migration is not completely understood, this orbital radius coincides (Lin et al. 1996) with the inner truncation point of the disk due to magnetic effects (Shu et al. 1994). As a result, Hot Jupiters generally enter $\sim 4$ day orbits with circumstellar disk material remaining outside. Additional bodies (rocky Earth-like planets and/or larger Neptune-like planets) can subsequently migrate into the vicinity, where they tend to lock into mean motion resonance with the Hot Jupiter. Although the disk acts only on the outer rocky body, both planets continue to migrate and interact.

The inward migration of these additional bodies, while the Hot Jupiter is stranded inside the inner disk edge, presents an interesting dynamical problem. Many outcomes are possible, including collisions between the planets, producing Earth-Jupiter systems in mean motion resonance, and accretion of planets onto the star. The relative frequency of these outcomes is studied here. If the resonant system survives, it becomes a candidate for observing transit timing variations (Agol et al. 2005, hereafter ASSC). If rocky planets are accreted by the Hot Jupiter, its mass would increase. Since these rocky bodies have higher metallicities, and densities, than the original object, the planetary density generally increases. This mechanism thus alters the mass-radius relationship for Hot Jupiters and can help explain the diversity of planetary properties in the current sample. In particular, if the rocky bodies are large enough, they can survive the impact (Anic et al. 2007) and increase the core mass of the Jovian planet. The observed planet HD149026b is inferred to 
have an exceptionally large core mass $M_{C} \sim 80 M_{\oplus}$ (Ikoma et al. 2006; Fortney et al. 2006) and may provide one example of this mechanism in action.

Working within this scenario, this Letter shows that a large fraction of inward migrating rocky planets collide with the Jovian planet, thereby allowing increases in core masses and metallicities. However, the collision rate decreases sharply for sufficiently high levels of eccentricity damping. If the Jovian planet has nonzero eccentricity, and/or smaller mass, the collision rate is lower for small damping rates, but persists for larger damping rates. For systems that experience planetary collisions, we determine the distributions of impact velocities.

This Letter focuses on collisions between rocky bodies and Hot Jupiters. A complete understanding of the planetary mass-radius relation requires many additional mechanisms, e.g., Ohmic dissipation in planetary atmospheres (Batygin \& Stevenson 2010, hereafter BS; Perna et al. 2010), which are beyond the scope of this work. In addition, Hot Jupiters display a range of spin-orbit alignments, measured through the Rossiter-McLaughlin effect (Fabrycky \& Winn 2009); some systems may have binary companions with inclined orbits so that planets are influenced by the Kozai effect (Wu et al. 2007). However, this Letter is limited to systems where stellar binary companions do not play a defining role.

\section{FORMULATION}

This Letter studies migration scenarios where the Hot Jupiter is already in place and a second body migrates inward. The most important parameters are the migration rate and eccentricity damping rate for the rocky planet, and the initial eccentricity and mass of the Jovian planet. Given that the star and the Hot Jupiter are much more massive than the rocky planet, the latter acts as a test particle (to leading order). If the rocky planet migrates sufficiently slowly, it generally becomes locked into mean motion resonance with the Hot Jupiter. Continued migration of the second body then pushes both planets inward, although this motion ceases if the second body reaches the inner edge of the disk (and this motion becomes ineffective if the second planet is too small). If migration ceases, the resulting pair of planets could survive in or near resonance. If the Hot Jupiter can be observed in transit, the second body can produce transit timing variations (ASSC). If migration occurs too quickly, the second planet passes through mean motion resonance (Quillen 2006; Ketchum et al. 2011) and will often experience a close encounter with the Hot Jupiter. The interaction event can result in either a collision between the planets (and assimilation of the rocky body) or the accretion of one planet (generally the smaller one) by the star. Planets are rarely scattered out of the solar system because the gravitational potential of the star (for a $\sim 4$ day orbit) is deeper than that of the Jovian planet (escape thus requires three-body effects). One goal of this work is to determine the branching ratios for the various outcomes-survival, accretion, scattering into the star-as a function of (Jovian) planetary mass and orbital eccentricity.

We approach this problem by performing direct numerical integrations of migrating planetary systems, i.e., we integrate the full set of 18 phase space variables for the three-body problem consisting of the star, Hot Jupiter, and a second migrating planet. These integrations are carried out using a Bulirsch-Stoer integration scheme. In addition to gravity, we include forcing terms that represent inward migration and eccentricity damping; these additional effects arise due to the forces exerted on the planet(s) by the circumstellar disk. However, we do not model the disk directly, but rather include forcing terms to model its behavior.

We consider simple disk models where the surface density and temperature distribution are power laws in radius,

$$
\Sigma(r)=\Sigma_{1}\left(\frac{r_{1}}{r}\right)^{p} \quad \text { and } \quad T(r)=T_{1}\left(\frac{r_{1}}{r}\right)^{q},
$$

where $\Sigma_{1}$ and $T_{1}$ are normalization constants. Here we take $r_{1}=1 \mathrm{AU}$, so the coefficients $\Sigma_{1}$ and $T_{1}$ correspond to values at $1 \mathrm{AU}$. The index $p=1-2$, where the intermediate value $p=$ $3 / 2$ arises for the minimum mass solar nebula (Weidenschilling 1977) and where recent observations suggest $p=0.9 \pm 0.2$ (Andrews et al. 2010). The normalization for the surface density has a range of values, with $\Sigma_{1} \approx 1500-4500 \mathrm{~g} \mathrm{~cm}^{-2}$ (Kuchner 2004). The power-law index of the temperature profile $q \approx 3 / 4$ for a viscous accretion disk (Pringle 1981) and a flat reprocessing disk (Adams \& Shu 1986), whereas $q \approx 1 / 2$ for a flared reprocessing disk (Chiang \& Goldreich 1997). The latter value is often used to describe the early solar nebula (Weidenschilling 1977).

The disk scale height $H=a_{S} / \Omega$, where $a_{S}$ is the sound speed, which is determined by the disk temperature profile. For a power-law temperature distribution, we obtain the form

$$
\frac{H}{r}=\left(\frac{H_{1}}{r_{1}}\right)\left(\frac{r}{r_{1}}\right)^{(1-q) / 2}
$$

where the scale height $H \approx 0.1 r$ at $r_{1}=1$ AU.

To account for planet migration, we assume that the semimajor axis of the outer planet decreases with time according to the ansatz

$$
\dot{a} / a=-1 / \tau_{a},
$$

where $\tau_{a}$ is the migration timescale, which varies with $a$. We assume that only the outer planet experiences torques from the circumstellar disk. Small planets (less massive than Saturn) cannot clear disk gaps, and migrate inward quickly through the process of Type I migration (Ward 1997). Larger bodies clear gaps and migrate more slowly. Planets are expected to experience a range of migration rates, depending on planet masses and disk properties. Estimates of the migration timescale for $a \sim 1$ AU typically fall in the range $10^{4}-10^{5} \mathrm{yr}$ (Goldreich \& Tremaine 1980; Papaloizou \& Larwood 2000). The migration timescale decreases with semi-major axis $a$ and can be modified by sub-Keplerian rotation (Adams et al. 2009). Since we must perform a large ensemble of simulations using effectively equivalent starting conditions, we adopt a relatively simple model of Type I migration.

The strength of Type I torques can be written in the form

$$
T_{I}=f_{1}\left(\frac{m_{P}}{M_{*}}\right)^{2} \pi \Sigma r^{2}(r \Omega)^{2}\left(\frac{r}{H}\right)^{2},
$$

where $m_{P}$ is the mass of the rocky planet and $f_{1} \approx 0.6$ is a dimensionless parameter (Ward 1997; Tanaka et al. 2002). For nearly Keplerian disks, the orbital angular momentum for a circular orbit is given by $J=m_{P}\left(G M_{*} r\right)^{1 / 2}$, and the migration timescale $\tau_{a}$ becomes

$$
\tau_{a}=\frac{J}{T_{I}}=\frac{1}{f_{1}}\left(\frac{M_{*}}{m_{P}}\right)\left(\frac{M_{*}}{\pi \Sigma r^{2}}\right)\left(\frac{H}{r}\right)^{2} \frac{1}{\Omega} .
$$

Using typical parameter values, we obtain the scaled result

$$
\tau_{a}=5.6 \times 10^{4} \mathrm{yr}\left(\frac{r}{r_{1}}\right)^{p-q+1 / 2}\left(\frac{m_{P}}{10 M_{\oplus}}\right)^{-1} .
$$




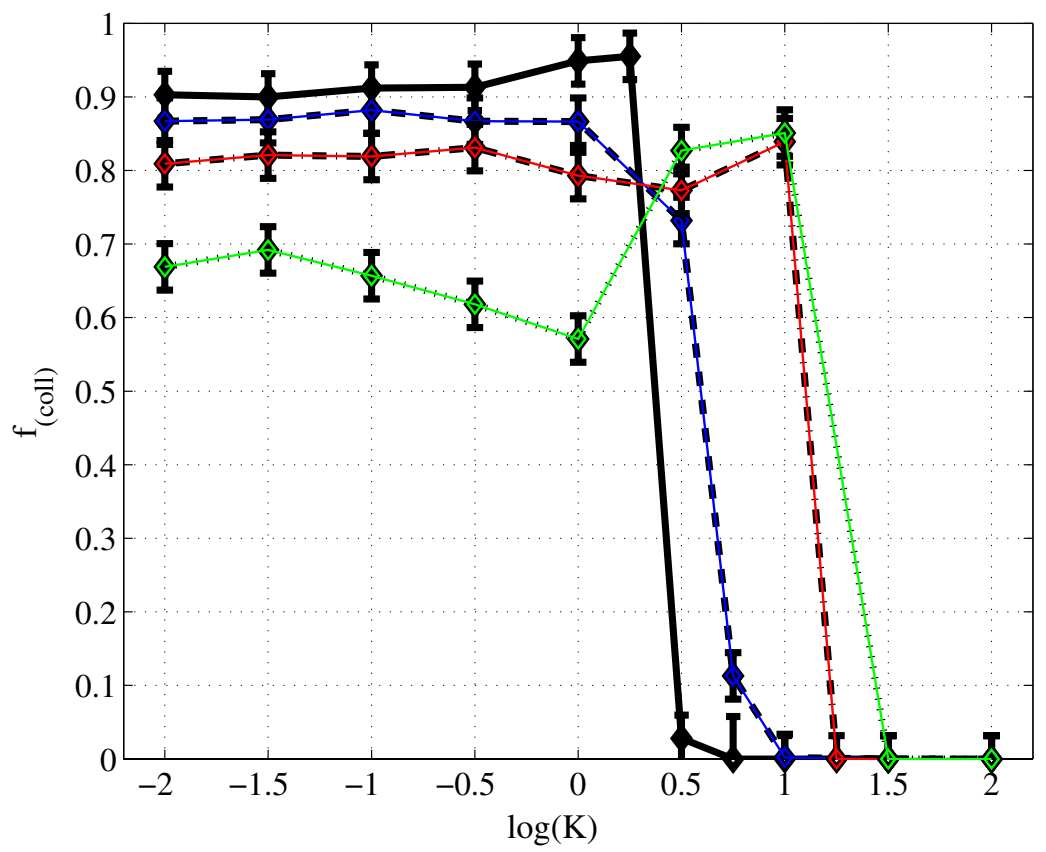

Figure 1. Collision fraction for rocky planets impacting Hot Jupiters vs. eccentricity damping parameter $K$. The curves correspond to varying initial eccentricity of the Jovian orbit: $e=0$ (black solid), $e=0.1$ (blue dashes), $e=0.2$ (red dot dashes), and $e=0.3$ (green dots).

(A color version of this figure is available in the online journal.)

We adopt the indices used to model the early solar nebula, $p=3 / 2$ and $q=1 / 2$, so the migration timescale is proportional to the orbital period,

$$
\tau_{a} \approx 56,000 P_{\mathrm{orb}}\left(m_{P} / 10 M_{\oplus}\right)^{-1}
$$

where the period is in years. The timescale $\tau_{a}$ thus decreases as the rocky planet moves inward, i.e., migration accelerates.

In addition to inward migration, circumstellar disks damp the orbital eccentricity $e$ of the migrating planet. This damping effect arises in almost all numerical simulations of the process (e.g., Kley et al. 2004), and can be parameterized through the ansatz

$$
\dot{e} / e=-1 / \tau_{e}=K(\dot{a} / a) \quad \text { so that } \quad \tau_{e}=\tau_{a} / K,
$$

where $\tau_{e}$ is the eccentricity damping timescale. For planets that are large enough to clear gaps, analytic calculations suggest that eccentricity can be excited through the action of disk torques (Goldreich \& Sari 2003; Ogilvie \& Lubow 2003), although multiple planet systems would be compromised if this were always the case (Moorhead \& Adams 2005). For smaller planets that remain embedded only eccentricity damping is expected. Given the uncertainties, we parameterize the eccentricity damping using Equation (8) and explore a wide range of the damping parameter $K$ such that $10^{-2} \leqslant K \leqslant 10^{2}$, where fully embedded planets are expected to have $K$ values at the high end of this range (Artymowicz 1993).

Note that this treatment implicitly assumes that the migrating planets are small enough so that they produce no back reaction on the disk. Since we are primarily interested in planetary cores in the mass range $m_{P}=1-30 M_{\oplus}$, this assumption is expected to be valid.

\section{RESULTS}

Using the formulation outlined above, we study the inward migration of rocky planets in planetary systems that contain a Hot Jupiter. The primary objective is to catalog the probabilities of the various outcomes, including survival, collisions, and accretion onto the star. A secondary goal is to determine the distribution of impact velocities for those cases that end in planetary collisions.

The parameter space for this study is large. For the sake of definiteness, the star has mass $M_{*}=1.0 M_{\odot}$ and the Jovian planet has starting semi-major axis $a=0.05 \mathrm{AU}$ ( $P_{\text {orb }} \approx 4$ days). The eccentricity of the giant planet varies over the range $0 \leqslant e \leqslant 0.3$ (these planets are expected to become tidally circularized, but only on much longer timescales). The rocky planet starts just outside the 5:1 mean motion resonance $(a \approx 0.15 \mathrm{AU})$, with small eccentricity $e=0.001$, and fixed mass $m_{P}=10 M_{\oplus}$; in this problem, the rocky planet acts like a test particle, so its mass cannot greatly affect the dynamics. The migration rate of the rocky planet varies with location, according to Equation (7); inside the disk edge ( $a \lesssim 0.05 \mathrm{AU}$ ), migration ceases. With these specifications, we consider the effects of varying the mass and eccentricity of the Jovian planet, and the eccentricity damping rate (through $K$ ) of the rocky planet. Since these systems are highly chaotic, a large ensemble of numerical experiments must be performed for each point in parameter space ( $\sim 1000$ independent realizations).

The main result from these simulations is the fraction of the trials that end with the two planets colliding. For a given migration rate, collisions represent the most common outcome provided that eccentricity damping is not too effective. These results are depicted in Figures 1 and 2, which show the fraction of collisions plotted versus the parameter $K$ that sets the strength of eccentricity damping for the rocky planet (Equation (8)). Figure 1 shows collision fractions for four choices of starting eccentricity for the Hot Jupiter, from $e=0$ to $e=0.3$. Figure 2 shows collision fractions for fixed starting eccentricity $e=0.2$ and three choices for the Hot Jupiter mass, $M_{P} / M_{J}=0.5$, 1 , and 2 . In both figures, each point shown corresponds to the fractions calculated from $N \sim 1000$ independent realizations of the starting conditions. The error bars $(\sim 1 / \sqrt{N})$ provide a crude measure of the uncertainties. 


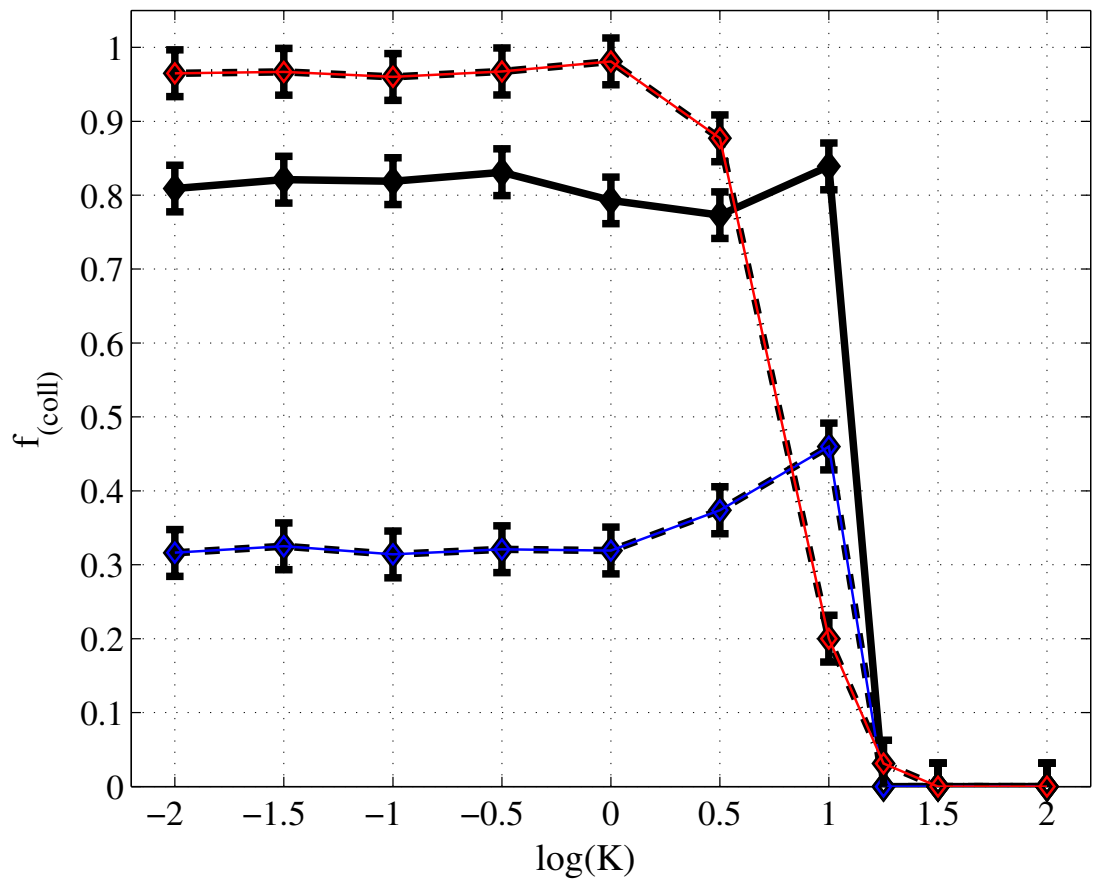

Figure 2. Collision fraction for rocky planets impacting Hot Jupiters vs. eccentricity damping parameter $K$. The curves correspond to varying masses of the Jovian planet: $0.5 M_{J}$ (red dot dashes, top), $1 M_{J}$ (black solid, middle), and $2 M_{J}$ (blue dashes, bottom).

(A color version of this figure is available in the online journal.)
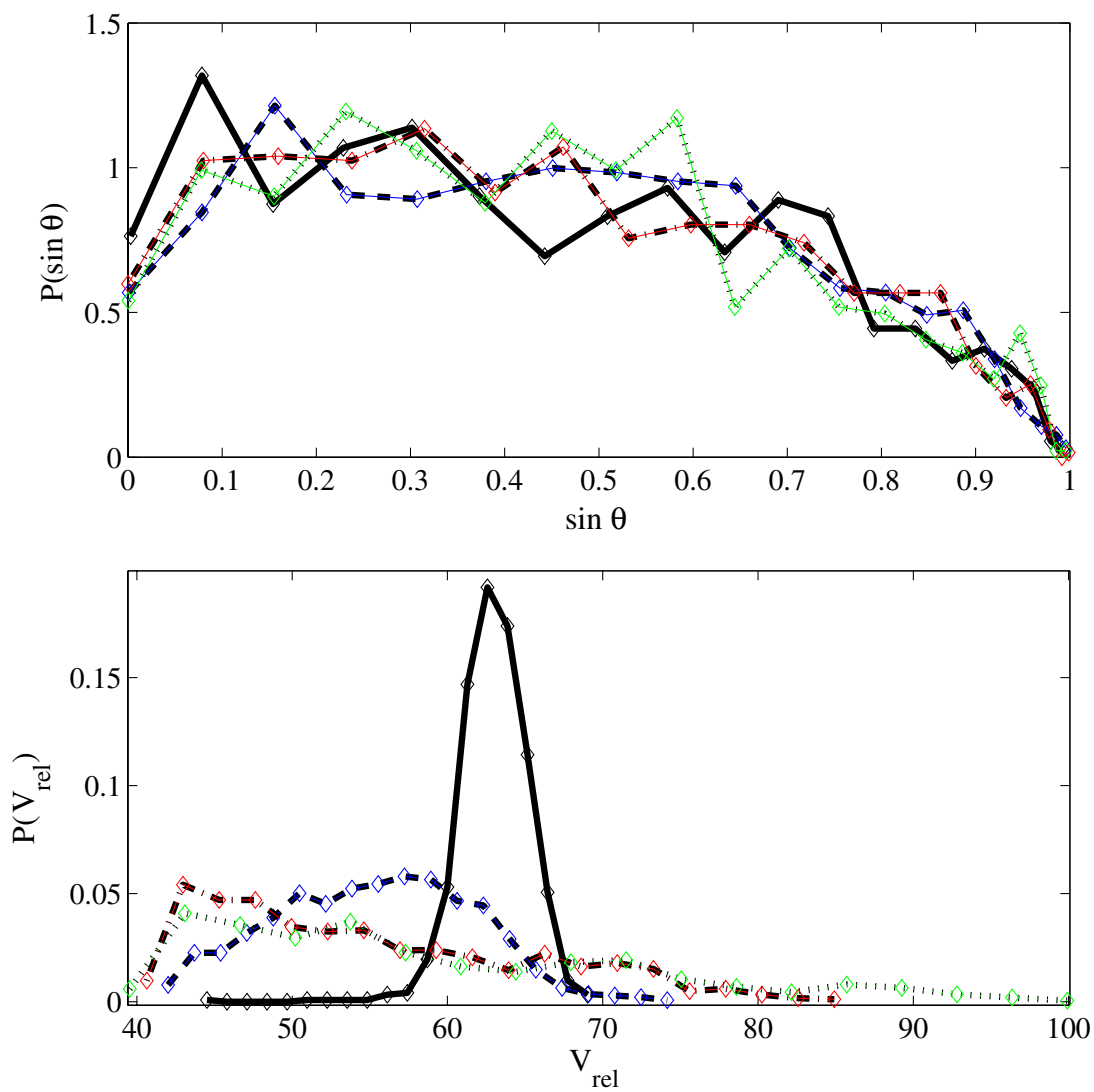

Figure 3. Distribution of impact velocities (for $K=1$ ). Top panel shows distributions of impact directions, specified by $\sin \theta$, where the angle $\theta$ is given by $\cos \theta=\hat{v} \cdot \hat{r}$. Bottom panel shows distributions of impact speeds. In both panels, distributions are shown for systems where the Jovian planet has initial eccentricity $e=0$ (black solid), $e=0.1$ (blue dashes), $e=0.2$ (red dot dashes), and $e=0.3$ (green dots).

(A color version of this figure is available in the online journal.) 
The results displayed in Figures 1 and 2 show a robust trend: for sufficiently weak eccentricity damping, $K<K_{C} \approx 10$, most simulations end with collisions between the planets. For stronger eccentricity damping, $K>K_{C}$, the collision fraction becomes negligible and nearly all of the systems survive (keeping both planets) over the entire range of integration times. Further, the critical level of eccentricity damping $\left(K_{C}\right)$ depends on the starting eccentricity and mass of the Hot Jupiter.

Larger eccentricities (for the Jovian orbit) allow collisions to occur in the face of greater eccentricity damping, following a trend of the approximate form $\log _{10} K_{C} \approx(3+10 e) / 4$ (from fitting). However, larger eccentricities combined with smaller $K$ values yield lower collision rates. In this regime, collision events are replaced (primarily) by accretion events (onto the star). The larger eccentricity of the Jovian planet provides the rocky planet with greater opportunity to pass by and enter the gravitational realm of the star. Similarly, larger masses for the Jovian planet allow collisions to occur for larger values of the eccentricity damping parameter. In addition, larger masses combined with smaller $K$ values lead to lower collision rates. In this case, the collision events are (again) replaced with accretion events. The larger mass of the Jovian planet can scatter the rocky planet before impact, and the scattering alters the orbit of the rocky planet enough to send it into the star (or, more rarely, eject the planet).

These results were obtained for a single migration rate; for faster (slower) migration, the outer planet is less (more) likely to lock into mean motion resonance and is more (less) likely to collide with the Jovian planet (Ketchum et al. 2011). We have performed additional simulations with faster migration (not shown) to confirm these trends.

For simulations that end in planetary collisions, the effect on the Jovian planet depends on the impact velocity of the rocky planet. Distributions of these impact velocities are depicted in Figure 3 (for eccentricity damping parameter $K=1$ ). The top panel shows distributions of the angle at which the incoming planet strikes the giant planet surface. This distribution is equivalent to the distribution of impact parameter $\varpi=R_{P} \sin \theta$. Collision dynamics depend on the impact speed $v_{\text {rel }}$, shown in the bottom panel of Figure 3 , and the escape speed $v_{\mathrm{esc}}=$ $\left(G M_{P} / R_{P}\right)^{1 / 2} \approx 37 \mathrm{~km} \mathrm{~s}^{-1}$ (for $M_{P}=1 M_{J}$ and $\left.R_{P}=1.4 R_{J}\right)$. In the limit $v_{\text {rel }} \gg v_{\text {esc }}$, the giant planet presents a circular target and the probability $P(\varpi) \propto P(\sin \theta)$ increases with impact parameter $\varpi$. In the limit $v_{\text {rel }} \ll v_{\text {esc }}$, gravity focuses incoming trajectories into nearly radial paths and the distribution peaks near $\varpi=0$. The calculated distribution is relatively flat, but falls with $\varpi$, which suggests significant gravitational focusing. This expectation is validated in the bottom panel, which shows that the impact speeds fall in the range $v \sim 40-100 \mathrm{~km} \mathrm{~s}^{-1}$, i.e., $v / v_{\text {esc }} \sim 1-3$. When the Jovian planet has nonzero eccentricity, the velocity distribution shows a broad peak near $v=50 \mathrm{~km} \mathrm{~s}^{-1}$. For systems with $e=0$, however, the distribution has a narrower peak near $v=65 \mathrm{~km} \mathrm{~s}^{-1}$. One reason for this difference is that the rocky planets migrate further inward (before colliding) when $e=0$, so they are deeper in the gravitational potential well of the star. Obtaining a greater dynamical understanding of this trend provides an interesting problem for future investigation.

\section{CONCLUSION}

This Letter explores the accretion of rocky planetary bodies by Hot Jupiters after they reach close-in orbits. The results show that collisions between planets are common when the eccentricity damping rate is sufficiently small, and rare otherwise. In approximate terms, collisions require the eccentricity damping parameter $K \leqslant K_{C} \approx 10$, where the threshold $K_{C}$ depends on the eccentricity and mass of the Jovian planet (Figures 1 and 2). The corresponding distributions of impact velocities for the collisions are shown in Figure 3.

These results have important implications for the diversity seen in the observational sample of Hot Jupiters: for large $K$ values, both planets usually survive, in resonance, and such systems can exhibit observable transit timing variations (ASSC). For small $K$ values, collisions are common whenever disks produce rocky bodies after a Hot Jupiter has migrated to its inner orbit. These collisions, in turn, can increase the core mass and the metallicity of the Jovian planet. Accretion onto the star and ejection are almost always rare.

The frequency of collisions is governed by the $K$ value, which depends on disk structure, viscosity, and the mass of the migrating rocky planet. Previous studies of planet-disk interactions generally find $K$ values of order unity for migrating planets that clear gaps (Kley et al. 2004), but $K \approx 10-30$ for smaller embedded planets (Artymowicz 1993). The outcomes thus depend on gap-clearing. For low-viscosity disks, planets clear gaps when their Hill sphere exceeds the disk scale height, $r_{H}>H$ (Crida et al. 2008; Papaloizou \& Terquem 2006), which requires $m_{P} \gtrsim 27 M_{\oplus}$ for the disk parameters used here. The gap does not need to be completely open to reduce the $K$ value below the threshold $K_{C}$. Nonetheless, relatively large rocky planets $\left(m_{P} \gtrsim 10-20 M_{\oplus}\right)$ are required for partial gap-clearing, reduced $K$ values, and hence collisions. Small planets with $m_{P} \lesssim 10 M_{\oplus}$ are expected to have $K>K_{C}$ and hence to avoid collision with high probability. In addition, incoming rocky bodies must survive the collision and reach the core to increase its mass; survival is expected when $m_{P} \gtrsim 1-10 M_{\oplus}$ (Anic et al. 2007). Both the occurrence of collisions and subsequent survival to reach the core thus require $m_{P} \gtrsim 10 M_{\oplus}$. Although this threshold mass should be determined more rigorously, these results show that larger rocky planets have more influence (per unit mass) than smaller ones.

In addition to increasing the core mass, accretion of rocky planets can affect the energy budget of giant planets. Figure 3 shows the distribution of impact speeds for rocky planets that collide with Hot Jupiters. This distribution indicates speeds $v \sim$ 40-100 $\mathrm{km} \mathrm{s}^{-1}$, so we consider a benchmark $v \sim 60 \mathrm{~km} \mathrm{~s}^{-1}$. With this speed, an accreting "superearth" planet with mass $m_{P}=10 M_{\oplus}$ deposits energy

$$
\Delta E=\frac{1}{2} m_{P} v^{2} \approx 1.1 \times 10^{42} \mathrm{erg}
$$

To put this energy increment into perspective, note that the binding energy of the Hot Jupiter $U=f G M_{P}^{2} / R_{P} \approx 1.6 \times$ $10^{43} \mathrm{erg}$ (using typical values $M_{P}=1 M_{J}, R_{P}=1.4 M_{J}$, and $f=3 / 5$ ). A single collision thus accounts for $\sim 7 \%$ of the binding energy of a Hot Jupiter. If we assume the energy $\Delta E$ is deposited deep within the planet, and slowly leaks out over time $\Delta t \sim 1 \mathrm{Gyr}$, the associated power increment $\Delta P \approx 3.5 \times 10^{18} \mathrm{~W}$, large enough to help inflate the planetary radius (BLL; BS). On the other hand, if the energy is deposited in the upper atmosphere of the planet, it quickly radiates away and cannot inflate the radius.

The results of this Letter pose a number of interesting problems for future work. To determine the number of accretion events (per Hot Jupiter), we need a better understanding of eccentricity damping rates for both migrating rocky planets and Hot Jupiters; we also need estimates for the number (and 
masses) of rocky planets produced after Hot Jupiter migration has occurred. When accretion events take place, we need to understand the energy deposition within the giant planet and the subsequent long-term transfer of heat/energy out of the planetary body. These issues, and others, will help explain the observed diversity in the properties of Hot Jupiters.

This work was supported by NSF grant DMS-0806756 from the Division of Applied Mathematics, NASA grant NNX11AK87G (F.C.A.), and NSF grant DMS-0907949 (A.M.B.).

\section{REFERENCES}

Adams, F. C., Cai, M. J., \& Lizano, S. 2009, ApJ, 702, L182

Adams, F. C., \& Shu, F. H. 1986, ApJ, 308, 836

Agol, E., Steffen, J., Sari, R., \& Clarkson, W. 2005, MNRAS, 359, 567 (ASSC)

Andrews, S. M., Wilner, D. J., Hughes, A. M., Qi, C., \& Dullemond, C. P. 2010, ApJ, 723, 1241

Anic, A., Alibert, Y., \& Benz, W. 2007, A\&A, 466, 717

Artymowicz, P. 1993, ApJ, 419, 166

Batygin, K., \& Stevenson, D. J. 2010, ApJ, 714, 238 (BS)

Bodenheimer, P., Laughlin, G., \& Lin, D. N. C. 2003, ApJ, 592, 555 (BLL)

Chiang, E. I., \& Goldreich, P. 1997, ApJ, 490, 368

Crida, A., Sándor, Z., \& Kley, W. 2008, A\&A, 483, 325

Fabrycky, D. C., \& Winn, J. N. 2009, ApJ, 696, 1230

Fortney, J. J., Saumon, D., Marley, M. S., Lodders, K., \& Freedman, R. S. 2006, ApJ, 642, 495
Goldreich, P., \& Sari, R. 2003, ApJ, 585, 1024

Goldreich, P., \& Tremaine, S. 1980, ApJ, 241, 424

Hatzes, A. P., Cochran, W. D., McArthur, B., et al. 2000, ApJ, 544, L145

Ikoma, M., Guillot, T., Genda, H., Tanigawa, T., \& Ida, S. 2006, ApJ, 650, 1150

Ketchum, J. A., Adams, F. C., \& Bloch, A. M. 2011, ApJ, 726, 53

Kley, W., Peitz, J., \& Bryden, G. 2004, A\&A, 414, 735

Kuchner, M. J. 2004, ApJ, 612, 1147

Laughlin, G., Crismani, M., \& Adams, F. C. 2011, ApJ, 729, L7

Lin, D. N. C., Bodenheimer, P., \& Richardson, D. C. 1996, Nature, 6575 , 606

Marcy, G. W., \& Butler, R. P. 1996, ApJ, 464, L147

Marcy, G. W., \& Butler, R. P. 2000, PASP, 112, 137

Mayor, M., \& Queloz, D. 1995, Nature, 378, 355

Moorhead, A. V., \& Adams, F. C. 2005, Icarus, 178, 517

Ogilvie, G., \& Lubow, S. 2003, ApJ, 587, 398

Papaloizou, J. C. B., \& Larwood, J. D. 2000, MNRAS, 315, 823

Papaloizou, J. C. B., \& Terquem, C. 2006, Rep. Prog. Phys., 69, 119

Perna, R., Menou, K., \& Rauscher, E. 2010, ApJ, 724, 313

Perryman, M. A. C. 2000, Rep. Prog. Phys., 63, 1209

Pringle, J. E. 1981, ARA\&A, 19, 137

Quillen, A. C. 2006, MNRAS, 365, 1367

Shu, F. H., Najita, J., Ostriker, E., et al. 1994, ApJ, 429, 781

Tanaka, H., Takeuchi, T., \& Ward, W. R. 2002, ApJ, 565, 1257

Udry, S., Fischer, D., \& Queloz, D. 2007, in Protostars and Planets V, ed. B. Reipurth, D. Jewitt, \& K. Keil (Tuscon, AZ: Univ. Arizona Press), 685

Ward, W. R. 1997, Icarus, 126, 261

Weidenschilling, S. J. 1977, Ap\&SS, 51, 153

Wu, Y., Murray, N. W., \& Ramsahai, J. M. 2007, ApJ, 670, 825 